\title{
Specificity of Alternative Splice Form Detection Using RT-PCR with a Primer Spanning the Exon Junction
}

Natalia Shulzhenko, Anna S. Smirnova, Andrey Morgun, and Maria Gerbase-DeLima Federal University of São Paulo (UNIFESP-EPM), São Paulo, Brazil

\footnotetext{
ABSTRACT

$A$ recently described strategy for splice variant specific detection by RT-PCR is based on the use of a primer spanning the junction between exons of the alternative splice form. However, this reaction may generate false-positive results in the presence of excess principal transcript. In this report, transcript variant 3 of T cell immune regulator gene 1 was used as a model to demonstrate a new method to ensure PCR specificity. Our approach permits the determination of detection specificity considering the full-length transcript amount. Furthermore, we demonstrated that the addition of a few molecules of a specific template dramatically increases the specificity of the reaction and allows for the detection of the alternative form, even in the presence of large amounts of the principal transcript. Competitor DNA for the alternative splice form is suggested as the specific template to achieve the detection specificity. Thus, we describe a simple strategy to avoid nonspecific amplifications for RT-PCR using a primer spanning the exon junction.
}

\section{INTRODUCTION}

According to recently reported genomic analyses, $40 \%-60 \%$ of human genes have alternative splice forms (1). Alternative splicing enables the generation of different mRNA isoforms from the same primary transcript and changes the protein product in $70 \%$ $80 \%$ of cases (2). Because different proteins produced from a single gene may perform distinct biological functions, alternative splicing is an important source of an organism's complexity, but it has not been well characterized for most genes. A new bioinformatics approach, based on identifying expressed sequence tags from the same gene, permits the prediction of candidate splices (3). Consequently, there is a growing interest in methods of alternative splice form verification.

Today, the most rapid and sensitive technique for mRNA detection is RTPCR. The conventional PCR strategy for the detection of splice variants is a simultaneous amplification of several templates with primers flanking the alternatively spliced region. This approach permits the estimation of the relative expression of the splice variants but does not allow for separate quantification of a particular transcript. Besides, amplification of a lowly expressed splice variant may be inhibited by competition with the more highly expressed form. In spite of these drawbacks, most investigators use this approach for mRNA isoform detection. In 1994, Yang and Le (4) suggested a new strategy for splice variant specific am- plification that has been actively used since 2001 (5-9). This strategy is based on the use of a primer spanning the specific exon-exon junction of the alternative splice form. However, the amplification with such a primer may generate false-positive results in the presence of excess principal transcript (10). Therefore, we developed a clear and simple strategy to delimit the detection specificity, and we propose a new approach to avoid nonspecific amplifications. In this report, this approach is illustrated by the specific amplification of a splice variant of $\mathrm{T}$ cell immune regulator gene 1 (TCIRG1).

TCIRG1 has three mRNA isoforms: transcript variant 1 , transcript variant 2 , and transcript variant 3 (GenBank ${ }^{\circledR}$ accession nos. NM_006019, NM 006053, and AF4975 $\overline{45}$, respectively). TCIRG1 contains 20 exons that are numbered in reference to transcript variant 1 . Transcript variants 1 and 2 use alternative transcription start sites but share the last 14 exons. Here we do not distinguish between these two isoforms and designate them as the principal transcript. Transcript variant 3 , which we recently found (manuscript in preparation), lacks exon 19. For the purpose of this study, it is designated as the alternative splice form.

\section{MATERIALS AND METHODS}

Cervical cancer and normal adjacent tissue specimens were collected from patients after we obtained their informed consent. The study was approved by the 
Ethics Committee of the Federal University of São Paulo. The tissue fragments were sheared with a HandiShear AC homogenizer (VirTis, Gardiner, NY, USA) in $0.8 \mathrm{~mL}$ TRIzOL ${ }^{\circledR}$ reagent (Invitrogen, Carlsbad, CA, USA) to extract total RNA. Reverse transcription was performed in a final volume of $50 \mu \mathrm{L}$ using $1 \mu \mathrm{g}$ of the isolated RNA, $400 \mathrm{U}$ SuperScript ${ }^{\mathrm{TM}}$ II $\mathrm{H}^{-}$reverse transcriptase, $10 \mu \mathrm{L}$ of reverse transcription buffer, $5 \mu \mathrm{L} 0.1 \mathrm{M}$ dithiothreitol solution, $10 \mu \mathrm{L} 10 \mathrm{mM}$ dNTPs (all from Invitrogen), $0.2 \mu \mathrm{g}$ oligo(dT) ${ }_{12-18}$ primer, and 50 U RNAguard ${ }^{\mathrm{TM}}$ RNase inhibitor (all from Amersham Biosciences, Uppsala, Sweden). This mixture was incubated at $42^{\circ} \mathrm{C}$ for $60 \mathrm{~min}$ and then at $65^{\circ} \mathrm{C}$ for $10 \mathrm{~min}$.

The primer sequences were: $5^{\prime}$-CTGGATGATGAAGAGGAGGCCGA-3', (S1); 5'-CCCTAGTCATCTGTGGCAGCGAA-3', (AS1); 5'-GGTCCAGGCCACGCTGGTGG-3', (S2); 5'-TTGTTCTGGAATTCCACCGGGC-3', (AS2); 5'-GTGCAGGAAGGCTGAGAGTCCC-3', (AS3). Competitor (CT) DNAs for the principal transcript and the alternative splice form were identical to the corresponding amplification product, but contained a 100-bp internal deletion (Figure 1) (11).

PCR was performed on $1 \mu \mathrm{L}$ of cDNA template using Ready-To-Go ${ }^{\mathrm{TM}}$ PCR beads (Amersham Biosciences) and $100 \mathrm{ng}$ of each primer. The amplification conditions were as follows: 40 cycles of denaturation at $94^{\circ} \mathrm{C}$ for $45 \mathrm{~s}$, annealing for $30 \mathrm{~s}$, and extension at $72^{\circ} \mathrm{C}$ for $30 \mathrm{~s}$ (PTC-200 ${ }^{\mathrm{TM}}$ thermal cycler; MJ Research, Waltham, MA, USA). The annealing temperature was $57^{\circ} \mathrm{C}$ for $\mathrm{S} 1$ and $\mathrm{AS} 1$ and $63^{\circ} \mathrm{C}$ for all other primer combinations. PCR products were separated by electrophoresis on ethidium bromide-stained $2 \%$ agarose gels, and the band intensities were analyzed using the Kodak Digital Science-EDAS 120 system (Eastman Kodak, Rochester, NY, USA). To purify PCR products, cDNA bands were extracted from the agarose gel using GFX ${ }^{\mathrm{TM}}$ PCR DNA and Gel Band Purification kit (Amersham Biosciences), following the manufacturer's instructions. The concentration of the purified PCR product was determined by comparing band intensities on the agarose gel with the Low DNA Mass ${ }^{\mathrm{TM}}$ Ladder (Invitrogen). The initial copy number of the product was calculated, considering that $1 \mathrm{~mol}$ of any substance contains 6 $\times 10^{23}$ molecules (Avogadro's number). Because a molar mass of a base pair is about $649 \mathrm{~g} / \mathrm{mol}, 1 \mathrm{fg}$ of $600 \mathrm{bp}$ cDNA corresponds to approximately 1,550 copies. The levels of the principal transcript in the tissue samples were evaluated by quantitative competitive (QC) RT-PCR (11)

\section{RESULTS}

\section{Primer Design}

Three primer pairs were used to amplify the TCIRG1 transcripts. Either sense primer S1 or S2, with the antisense AS1, simultaneously amplified the principal transcript and the alternative splice form (Figure 1). For a separate amplification of these two transcripts, the sense primer S2 was combined with the principal transcript specific antisense AS3 or with the alternative splice form specific antisense AS2 (Figure 1). AS2 spanned the junction between exons 18 and 20, with the 18 bases at the $5^{\prime}$ end being reverse complementary to exon 20 , and the four bases at the $3^{\prime}$ end, reverse complementary to exon 18 . However, a primer spanning the exon junction can nonspecifically anneal to the principal transcript if the latter is present in excess (Figure 1).

\section{Determination of Detection Specificity}

A cDNA sample that contained the principal transcript was amplified with $\mathrm{S} 2$ and AS1, and the band that corresponded to the principal transcript fragment was purified from the gel. After its quantification with the Low DNA Mass Ladder, the fragment was serially diluted 10 -fold from $13.6 \times 10^{9}$ up to $13.6 \times$ $10^{1}$ copies. Re-amplification of the product with the same primers yielded a band of the expected size at all the dilutions (Figure 2A). The re-amplification of the principal transcript fragment with S2 and spanning the exon junction AS2 generated a product corresponding in size to that of the alternative splice form, at dilutions up to $13.6 \times 10^{3}$ copies (Figure 2B). This indicated a nonspecific annealing of the AS2 to the principal transcript. Amplifications of 2-fold dilutions of the principal transcript fragment from $13.6 \times 10^{3}$ copies, with $\mathrm{S} 2$ and spanning the exon junction AS2, demonstrated that the highest copy number of the principal transcript that did not lead to nonspecific amplification was approximately 1700 copies. This copy number was considered a limit of the detection specificity in the absence of a specific template.

Next, we determined how the specific template amount would influence the limit of the detection specificity. We used CT for the alternative splice form as the specific template. The CT sequence was equal to that of the alternative splice form amplification product obtained with S2 and AS2 but contained a 100-bp internal deletion (Figure 1). We co-amplified serial dilutions of the
Figure 1. Schematic representation of TCIRG1 principal transcript, alternative splice form, and its CT. The localization of PCR primers is indicated. Primer combinations and length of resulting PCR fragments are listed beside each transcript. An asterisk $(*)$ indicates a possible nonspecific amplification.

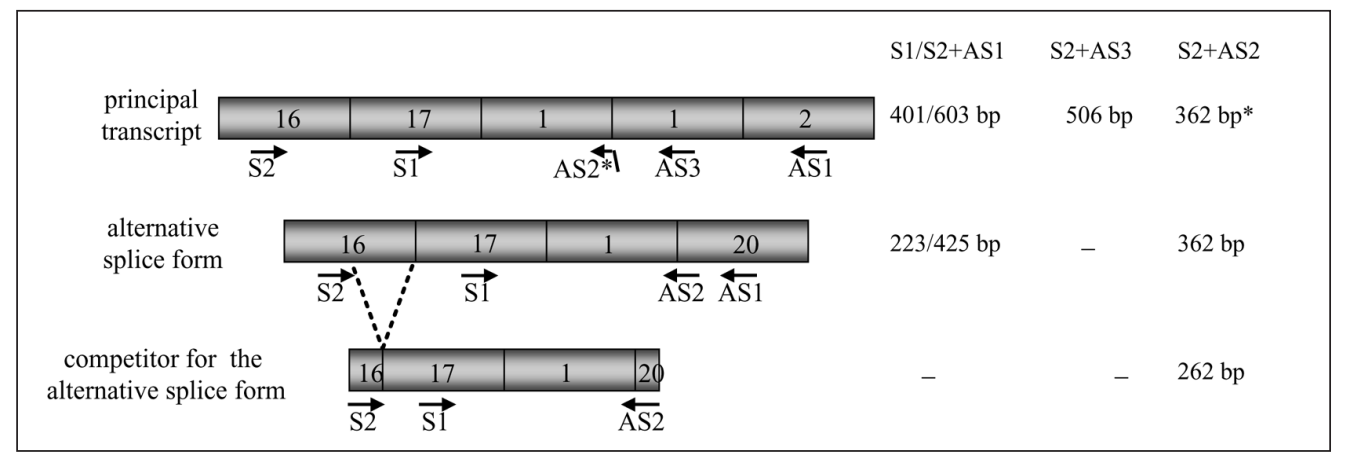




\section{Research Report}

principal transcript fragment and fixed amounts (36 or 72 copies) of the CT with $\mathrm{S} 2$ and spanning the exon junction AS2. The product size difference permitted us to distinguish between specific and nonspecific bands and to find the limit of the detection specificity. In the presence of 36 copies of CT (Figure $2 \mathrm{C})$, the nonspecific amplification of 17,000 copies of the principal transcript was not observed (Figure 2C, lane 3). The specificity limit in the presence of 72 CT copies increased to 34,000 copies of the principal transcript (data not shown). Taking together, the specificity limits defined in the presence of 0 , 36, and 72 CT copies, we observed a linear relation between the amount of the principal transcript and the amount of the specific transcript necessary to provide the detection specificity (Figure 2D). The combinations of transcript amounts above the curve generate specific amplifications, while those beneath the curve generate concomitant nonspecific amplifications.

\section{Specific Detection of the Alternative Splice Form in Samples}

To demonstrate our strategy for the specific detection of the alternative splice form in the presence of the excess of the principal transcript, we selected an uterine cervix cDNA sample that contained a small amount of the alternative splice form, detectable with S1 and AS1, and about 1700 copies of the principal transcript (Figure 3, lane 1). The principal transcript level was determined by QC RT-PCR (11). We then added 15,300 copies of the principal transcript fragment per $1 \mu \mathrm{L}$ of the sample to artificially generate the highest level of the principal transcript that we had observed in uterine cervix cDNA samples
(17,000 copies in $1 \mu \mathrm{L}$ cDNA). This resulted in no detection of the alternative splice form by amplification with S1 and AS1 (Figure 3, lane 2). The amplification of the sample with the addition of the principal transcript fragment with S2 and spanning the exon junction AS2 yielded a band that could represent the sum of specific and nonspecific amplifications (Figure 3, lane 3). Using the equation shown in Figure 2D, the copy number of CT for alternative splice form necessary to inhibit the nonspecific amplification of the principal transcript in the sample was 36 . When we added this amount of the CT to the reaction, amplification with $\mathrm{S} 2$ and AS2 yielded two bands that corresponded to the CT and to the alternative splice form (Figure 3, lane 4). Thus, we could specifically detect the alternative splice form in spite of its low abundance and excess of the principal transcript.

We developed a strategy to be followed when the amplification of excess principal transcript with the primer spanning the exon junction generates nonspecific amplification: (i) determination of the limit of the detection specificity in serial dilutions of the principal transcript in the absence of the specific template; (ii) measurement of the principal transcript amount in samples of interest; (iii) comparison of the principal transcript amount in samples of interest with the specificity limit in the absence of the specific template. If the principal transcript amount does not exceed the specificity limit, then the detection specificity of the alternative splice form in the samples is guaranteed, and amplification with primer spanning the exon junction may

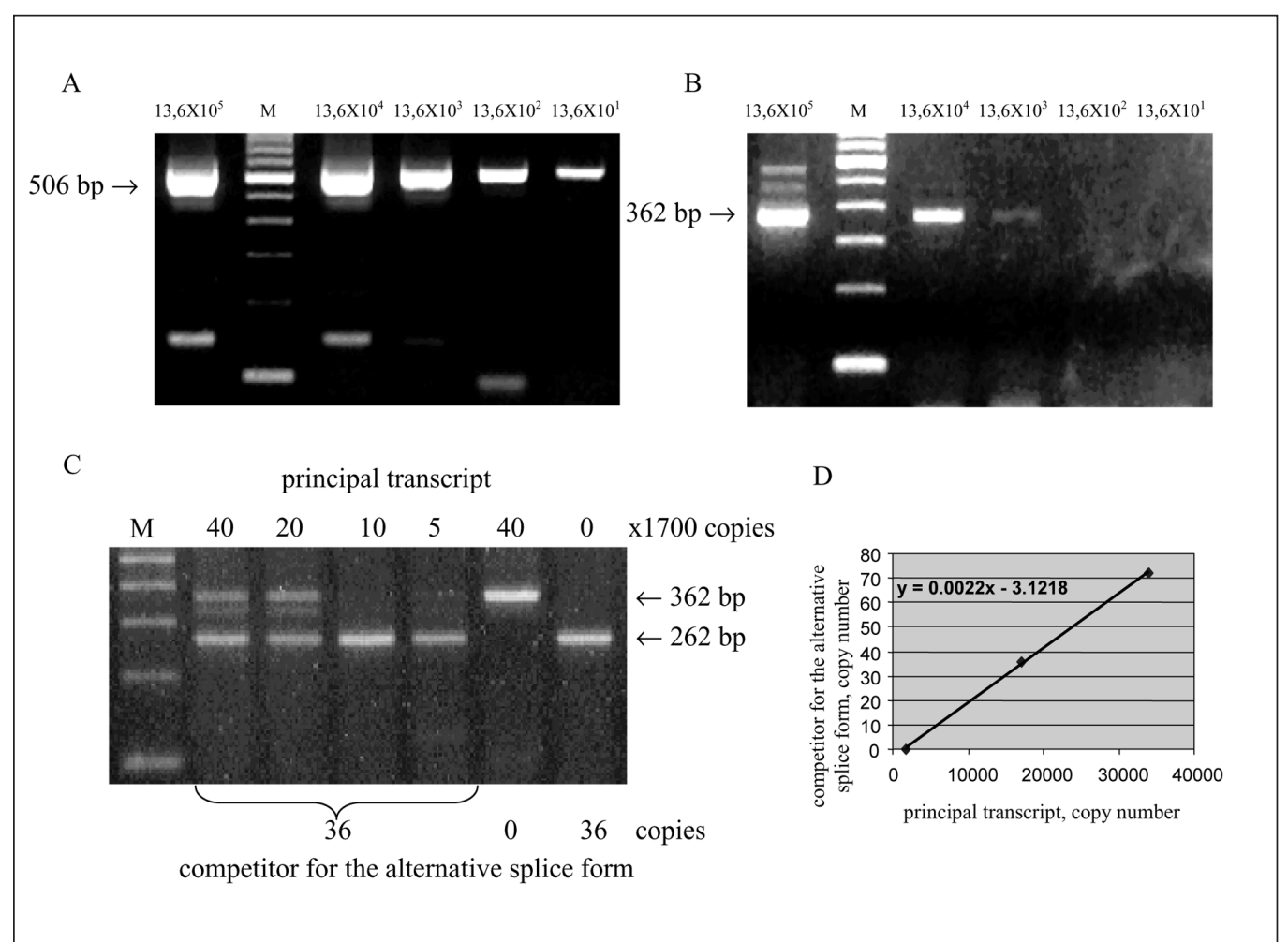

Figure 2. Determination of detection specificity. Re-amplificaton of the serial dilutions of the principal transcript fragment with primers S2 and AS1 flanking the alternative splice region (A) and with primers S2 and spanning the exon junction AS2 (B). (A and B) The initial number of copies of the principal transcript fragment in each reaction is indicated above each lane. Products with sizes different from those indicated are probably derived from the nonspecific annealing of the primers within the fragment. Lane M is a 100-bp molecular marker (Invitrogen). (C) Co-amplification of serial dilutions of the principal transcript fragment (product size, $362 \mathrm{bp}$ ) and 36 copies of the alternative splice form CT (product size, 262 bp) with primers S2 and AS2. The initial number of copies of the principal transcript fragment in each reaction is indicated above each lane; the number of the CT copies is beneath each lane. Lane M is a 100-bp molecular marker (Invitrogen). (D) The number of copies of the $\mathrm{CT}$ for the alternative splice form (y) necessary to provide the detection specificity in relation to the number of copies of the principal transcript $(\mathrm{x})$. Each point was reproduced in five independent experiments. 


\section{Research Report}

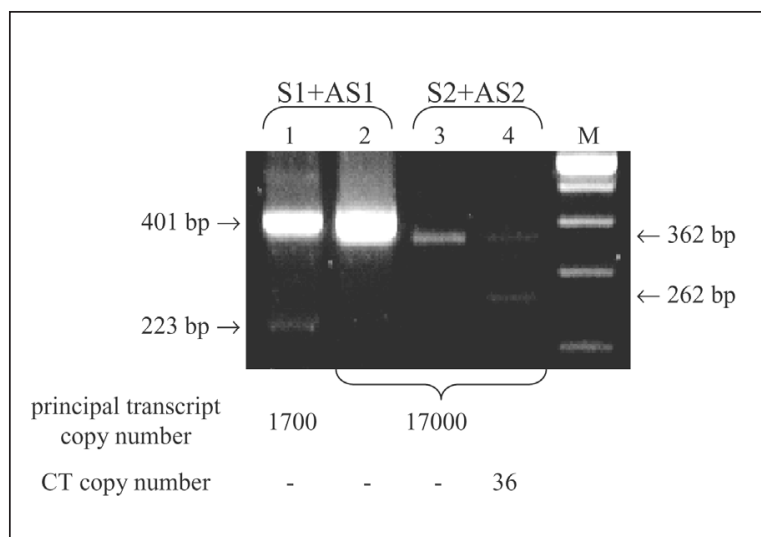

Figure 3. Alternative splice form detection in the presence of excess principal transcript. Lane 1, amplification of a sample containing 1700 copies of the principal transcript (product size, $401 \mathrm{bp}$ ) and a little amount of the alternative splice form (product size, $223 \mathrm{bp}$ ) with primers S1 and AS1 flanking the alternative splice region; lane 2, the same experiment as in lane 1 but with addition of 15,300 copies of the principal transcript fragment; lane 3, amplification of the sample with the addition of 15,300 copies of the principal transcript fragment with primers S2 and spanning the exon junction AS2 (product size, $362 \mathrm{bp}$ ); lane 4 , the same experiment as in lane 3 but with the addition of 36 copies of the CT for the alternative splice form (262 bp). Lane M is a $100-\mathrm{bp}$ molecular marker, and the lowest band corresponds to $200 \mathrm{bp}$. be performed without restrictions. If the principal transcript amount exceeds the specificity limit, then skip to the following steps of the strategy. (iv) Amplification of serial dilutions of the principal transcript with fixed amounts of CT for the alternative splice form and construction of the curve defining the relationship between the specific template amount and the specificity limit; $(v)$ calculation of the alternative splice form $\mathrm{CT}$ amount necessary to provide the specific amplification of the alternative splice form; (vi) amplification of the samples with the CT amount to separate the ones that contain the alternative splice form; and (vii) optimization of the CT amount to quantify the transcript.

\section{DISCUSSION}

The specificity of splice variant detection using RT-PCR with a primer spanning the exon junction depends on many factors, such as primer design, amplification conditions, and the fulllength and alternatively spliced transcript amounts. In the first study of this technique, the primer had three unique overhang bases across the splice site (4). However, a possible homology between the bases in the wild-type and alternative splice junction may require the extension of the base number. Dependence of the detection specificity on the primer design strategy was explored by Williams et al. (10). In our study, the re- 
verse primer designed for amplification of the TCIRG1 alternative splice form spanned the junction between exons 18 and 20 with four bases overhanging across the splice site, and there was no sequence homology between exons 18 and 19 of the gene. It is possible that nonspecific amplification with the primer spanning the exon junction is provided by the annealing of the whole primer with the formation of a hairpin in the principal transcript and/or to the annealing of only the primer's 3 '-end bases overhanging across the splice site.

A common way to determine the detection specificity is to amplify an excess amount of one mRNA isoform with primers specific for the other $(5,6,8)$. On the other hand, Vandenbroucke et al. (7), working with realtime RT-PCR, suggested the comparison of amplification plots resulting from serial dilutions of the shorter form, in the absence and in the presence of the full-length transcript. Most studies mention that specific amplification with a primer spanning the exon junction required extensive optimization of the reaction conditions, which results in the need for high annealing temperatures [e.g., $\left.68^{\circ} \mathrm{C}(6,8,10)\right]$ or additives $(7,9)$. Although these procedures increase the detection specificity, they are usually accompanied by a decrease in the amplification efficiency (12).

This study describes a new method to ensure the specificity for RT-PCR using a primer spanning the exon junction. We found that the nonspecific amplification depends on the principal transcript amount and suggest that the determination of the specificity limit considering the principal transcript amount could help to avoid unnecessary modifications of the reaction conditions. Optimization is only necessary when the principal transcript amount in samples of interest exceeds the specificity limit. Our suggestion to improve the detection specificity is to add the CT for the alternative splice form, whose amount may be calculated using an equation. This equation is obtained from the amplification of known amounts of the principal transcript and CT for the alternative splice form and the determination of the specificity limit at various points. The construction of the curve that separates the areas of the specific and nonspecific amplification helps to visualize the interrelation between the templates' amounts.

In our case, the specificity limit increased 10-fold with the addition of only 36 copies of the CT for the alternative splice form (Figure 2D). This increased limit was close to the highest amount of the principal transcript we have observed in the samples. Thus, we could specifically detect the alternative splice form by co-amplifying all our samples with the same CT amount. As illustrated in Figure 3, our strategy permitted the detection of the splice variant, even in the case when the sample contained the maximum amount of the principal transcript we had observed in the samples and a little amount of alternative splice form that was undetectable with primers flanking the splice region.

We have also successfully applied this strategy for the detection of a Fas isoform with a deletion of exon 6 and soluble isoforms of the CTLA-4 and PD-1 (data not shown). In all these cases, the principal transcript amount in the samples of interest did not exceed the specificity limit determined in the absence of the specific template (see step iii of the strategy).

This strategy can also be applied in real-time PCR, using the primer spanning the exon junction of the alternative splice form and the fluorescent probe in the region lacking in the CT. Thus, our approach may be widely used for the specific detection of splice variants by RT-PCR.

\section{ACKNOWLEDGMENTS}

This research was supported by Fundação de Amparo à Pesquisa do Estado de São Paulo (FAPESP) and Conselho Nacional de Pesquisa (CNPq). A.S.S. is the recipient of a Coordenação de Aperfeiçoamento de Pessoal de Nivel Superior (CAPES) fellowship. N.S., A.S.S., and A.M. contributed equally to this work.

\section{REFERENCES}

\footnotetext{
1.International Human Genome Sequencing Consortium. 2001. Initial sequencing and analysis of the human genome. Nature
}

409:860-921.

2.Modrek, B., A. Resch, C. Grasso, and C. Lee. 2001. Genome-wide detection of alternative splicing in expressed sequences of human genes. Nucleic Acids Res. 29:2850-2859.

3.Modrek, B. and C. Lee. 2002. A genomic view of alternative splicing. Nat. Genet. 30:13-19

4.Yang, D. and J. Le. 1994. Targeted amplification of alternatively spliced transcripts of major histocompatibility complex class I heavy chain. J. Immunol. Methods 176:265-270.

5.Goel, A., P. Seth, and S.S. Chauhan. 2001 Specific amplification of mRNA splice variants by RT-PCR. BioTechniques 30:944-948.

6.Mankertz, A. and B. Hillenbrand. 2001 Replication of porcine circovirus type 1 requires two proteins encoded by the vital rep gene. Virology 279:429-438.

7.Vandenbroucke, I.I., J. Vandesompele, A.D. Paepe, and L. Messiaen. 2001. Quantification of splice variants using real-time PCR Nucleic Acids Res. 29:e68.

8.Wellmann, S., T. Taube, K. Paal, V. Graf, H. Einsiedel, W. Geilen, G. Seifert, C. Eckert, et al. 2001. Specific reverse transcriptionPCR quantification of vascular endothelial growth factor (VEGF) splice variants by LightCycler technology. Clin. Chem. 47:654660.

9.Wong, Y.W., G.M. Sia, and H.P. Too. 2002 Quantification of mouse glial cell-line derived neurotrophic factor family receptor alpha 2 alternatively spliced isoforms by real time detection PCR using SYBR Green I. Neurosci. Lett. 320:141-145.

10.Williams, D.M., S. Koduri, Z. Li, W.D. Hankins, and I. Poola. 1999. Primer design strategies for the targeted amplification of alternatively spliced molecules. Anal. Biochem. 271:194-197.

11.Shulzhenko, N., A. Morgun, C.F. Rampim M. Franco, D.R. Almeida, R.V. Diniz, A.C. Carvalho, and M. Gerbase-DeLima. 2001. Monitoring of intragraft and peripheral blood TIRC7 expression as a diagnostic tool for acute cardiac rejection in humans. Hum. Immunol. 62:342-347.

12.Kovarova, M. and P. Draber. 2000. New specificityand yield enhancer of polymerase chain reactions. Nucleic Acids Res. 28:e70.

Received 20 November 2002; accepted 26 March 2003.

Address correspondence to:

Dr. Natalia Shulzhenko

Division of Immunogenetic

Pediatrics Department

Federal University of São Paulo

Rua Napoleão de Barros, 1038

04024-003 São Paulo, SP, Brazil

e-mail:anemorgun@hotmail.com 